\title{
Structural relaxation in orthoterphenyl: a schematic mode-coupling-theory model analysis
}

\author{
A.P. Singh ${ }^{\text {a }}$, G. Li ${ }^{\text {b }}$, W. Götze ${ }^{\text {a,* }}$, M. Fuchs ${ }^{\text {a }}$, T. Franosch ${ }^{\text {a }}$, H.Z. Cummins ${ }^{\text {b }}$ \\ a Physik-Department, Technische Universität München, 85747 Garching, Germany \\ b Department of Physics, City College, CUNY, New York, NY 10031, USA
}

\begin{abstract}
Depolarized light scattering spectra of orthoterphenyl showing the emergence of the structural relaxation below the oscillatory microscopic excitations are described by solutions of a schematic mode-coupling-theory model.
\end{abstract}

\section{Introduction}

Structural relaxation in glass forming liquids has been studied in great detail. Typically spectra are measured in the supercooled state for temperatures $T$ near the glass transition temperature $T_{\mathrm{g}}$ focusing on frequencies lower than, say, $10 \mathrm{MHz}$. However, it has been shown in many recent experiments that structural dynamics can be observed also for higher temperatures, even above the melting temperature $T_{\mathrm{m}}$. The relaxation spectra then show up in a frequency window just below the band of microscopic excitations, which is located near and above $1 \mathrm{THz}$. For example, the depolarized light scattering spectra of orthoterphenyl (OTP) exhibit a non-exponential $\alpha$-process within the $\mathrm{GHz}$ band for $T=T_{\mathrm{m}}+50 \mathrm{~K}[1,2]$.

The evolution of the structural relaxation upon cooling is the theme of the mode-coupling-theory

\footnotetext{
${ }^{*}$ Corresponding author. Tel.: 4989 28912360; fax: 4989 28914642; e-mail:wg@physik.tu-muenchen.de.
}

(MCT) of the glass transition [3]. This theory describes the dynamics of a liquid by a set of non-linear equations of motion, which use the equilibrium structure functions as input. Structural relaxation is obtained as anomalous dynamics, caused by a bifurcation singularity at some critical temperature $T_{\mathrm{c}}$, which is located typically between $T_{\mathrm{g}}$ and $T_{\mathrm{m}}$. Usually, data interpretation within MCT is based on universal results, obtained by solving the MCT equations asymptotically for $T$ close to $T_{\mathrm{c}}$. It was shown in Ref. [1] that a major part of the OTP-data could be described by using the universal MCT results for the $\alpha$ - and $\beta$-process. In the same frame neutron scattering spectra could be explained as is shown in Ref. [4] and papers quoted therein.

The shape of the $\alpha$-peak is not a universal feature of structural relaxation. It depends on the details of the microscopic structure and it is different for different probing variables [3]. The universal results of the MCT deal with relaxation only, and thus they cannot be used to discuss the crossover from relaxation at low frequencies to oscillations 
at higher frequencies. These shortcomings in the application of universal MCT results can be overcome to some extent by the application of schematic MCT models, as was shown recently in a discussion of the light scattering spectra of glycerol [5]. Such models truncate the microscopic MCT equations so that the universal features remain intact and that e.g. some of the oscillatory dynamics can still be described. Thereby, one can get solutions for the analysis of spectra which cover huge windows and which do not rely on asymptotic formulas. In this paper the MCT model of Ref. [5] shall be used to interpret the light scattering spectra of OTP $\left(T_{\mathrm{m}}=329 \mathrm{~K}, T_{\mathrm{g}}=244 \mathrm{~K}\right)$ from Ref. [1] for frequencies between 0.04 and $400 \mathrm{GHz}$ and temperatures between 225 and $395 \mathrm{~K}$.

\section{Schematic mode-coupling model}

The model to be used assumes that the structural relaxation of density fluctuations can be modeled by a single oscillator equation with a retarded friction function $m(t)$ :

$$
\begin{aligned}
& \partial_{t}^{2} \Phi(t)+v \partial_{t} \Phi(t)+\Omega^{2} \Phi(t) \\
& \quad+\Omega^{2} \int_{0}^{t} m\left(t-t^{\prime}\right) \partial_{t \prime} \Phi\left(t^{\prime}\right) d t^{\prime}=0
\end{aligned}
$$

The time-dependent friction $m(t)$ is calculated self-consistently from a non-linear functional in the autocorrelator $\Phi(t)$ of the oscillator variable

$$
m(t)=v_{1} \Phi(t)+v_{2} \Phi(t)^{2} .
$$

The coupling vertices $v_{\mathrm{i}}$ in microscopic MCT equations are known regular functions of the thermodynamic state variables [3]. In the schematic model the vertices are considered as coupling constants which vary smoothly with control variables like $T$. Different experimental techniques probe glassy dynamics in a different manner. Here it is assumed that a second correlator $\Phi_{\mathrm{s}}(t)$, or its susceptibility $\chi_{s}^{\prime \prime}(\omega)$, describes the variable probed by the depolarized light scattering spectra as a function of frequency $\omega$. The correlator $\Phi_{\mathrm{s}}(t)$ obeys an equation analogous to Eq. (1) and couples to the structural relaxation in its friction function via

$m_{\mathrm{s}}(t)=v_{\mathrm{s}} \Phi(t) \Phi_{\mathrm{s}}(t)$.
Thus, the model is specified by four oscillator parameters $\left(\Omega, v, \Omega_{\mathrm{s}}, v_{\mathrm{s}}\right)$ quantifying the microscopic excitations in the $\mathrm{THz}$ regime which will be taken as $T$-independent, and by three equilibrium state control parameters $\left(v_{1}, v_{2}, v_{\mathrm{s}}\right)$ whose regular $T$-dependence drives the spectacular changes of the spectra.

\section{Results}

Fig. 1 reproduces the susceptibility spectra measured by depolarized light scattering in OTP [1] and the fits using $\chi_{\mathrm{s}}^{\prime \prime}(\omega)$ from the schematic model. The trivial temperature variations of the data due to the Bose factor had been eliminated already in Ref. [1]. The spectra and corresponding fits cover frequency windows larger than three decades and temperatures from above the melting temperature $T_{\mathrm{m}}=329 \mathrm{~K}$ down to below the glass transition temperature $T_{\mathrm{g}}=244 \mathrm{~K}$. The $\alpha$-relaxation peak, observed at high temperatures, moves out of the experimental window below $T=310 \mathrm{~K}$. The excitations around $1 \mathrm{THz}$ show temperature dependence. Nevertheless, the oscillator parameters of the fit have been used as $T$-independent: $\Omega / 2 \pi=1.81 \mathrm{THz}, v=0, \Omega_{\mathrm{s}} / 2 \pi=0.14 \mathrm{THz} \quad$ and $v_{\mathrm{s}} / 2 \pi=0.44 \mathrm{THz}$. Obviously, the simple model of two microscopic oscillations does not suffice to fit the high frequency part of the spectra. We have restricted the fit to frequencies below $400 \mathrm{GHz}$.

The temperature change of the theoretical spectra is caused by the smooth drifts of the coupling vertices shown in Fig. 2. A rather simple path with a $T$-independent parameter $v_{1}=0.87$ was chosen. Coupling constants $v_{2}$ and $v_{\mathrm{s}}$, which increase smoothly with decreasing temperature drive the system through the MCT bifurcation singularity, which is located at $T_{\mathrm{c}} \approx 280 \mathrm{~K}$. The predicted universal features of the MCT spectra close to $T_{\mathrm{c}}$ [3] are determined by the exponent parameter $\lambda$, which equals $\lambda=0.735$ for this path.

\section{Discussion}

Fig. 1 verifies that the model captures the crossover from oscillatory microscopic dynamics near 


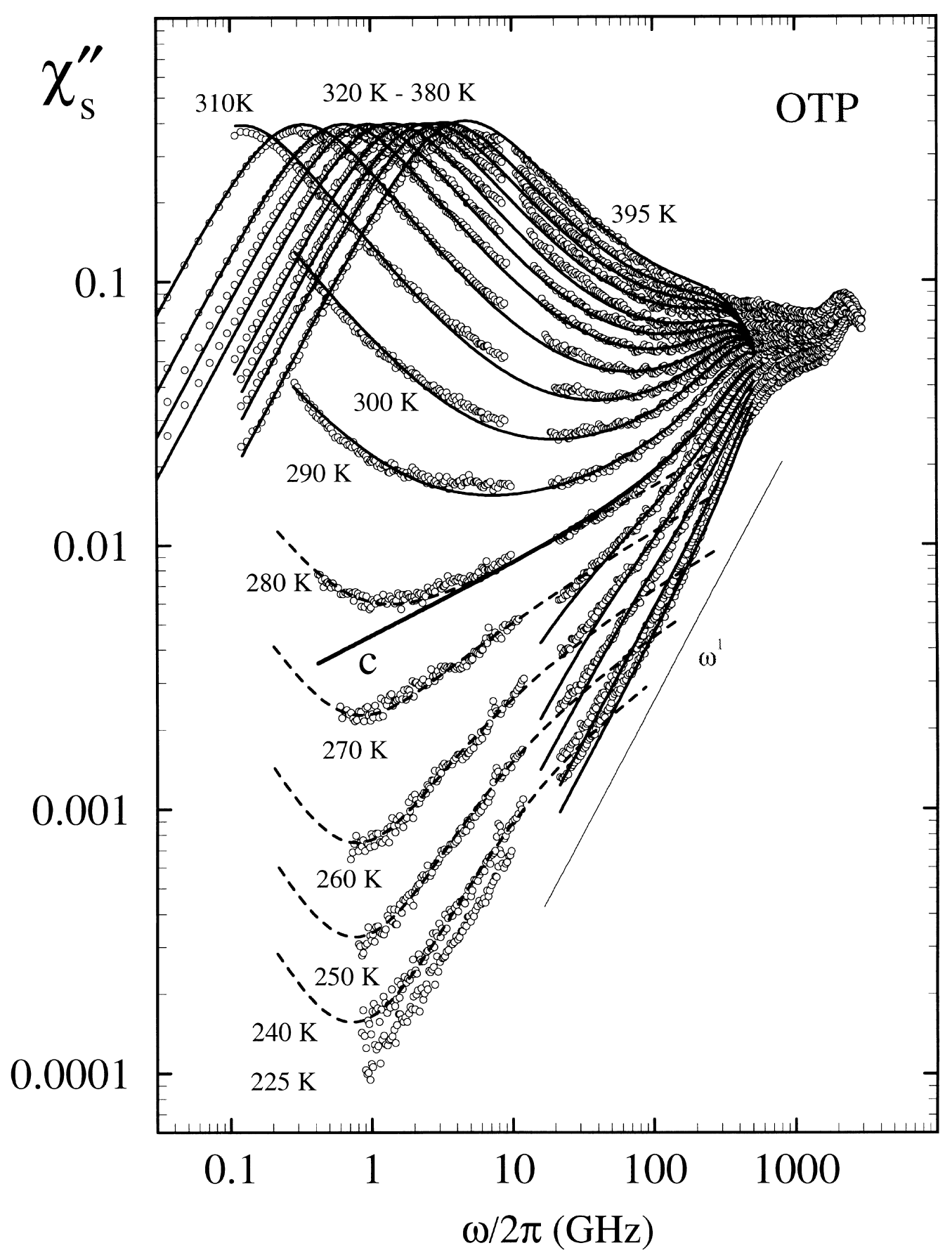

Fig. 1. Measured susceptibility spectra for OTP from Ref. [1] (open circles). The thin straight line indicates a white noise spectrum, $\chi_{\text {white }}^{\prime \prime}(\omega) \propto \omega$. The full lines are MCT solutions for the schematic model Eq. (1)-Eq. (3) with model parameters described in the text and in Fig. 2. The dashed lines show fits for intermediate frequencies with the asymptotic scaling law of the extended MCT which are discussed in Section 4 and in Ref. [1]. 


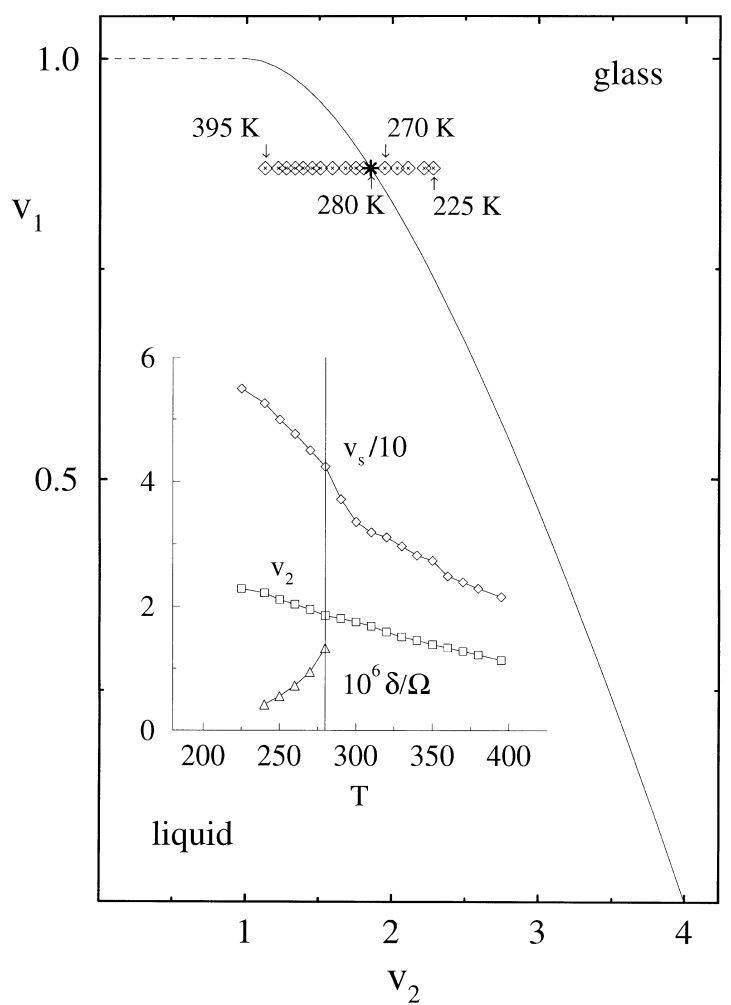

Fig. 2. Coupling constants for the schematic-model fits of Fig. 1. The liquid-glass transitions for the model occur either at the dashed line joining the $\left(v_{1}, v_{2}\right)$ points $(1,0)$ and $(1,1)$ or at the full parabola line joining $(1,1)$ and $(0,4)$. The diamonds with dots mark the $v_{1}-v_{2}$-pairs, chosen for the solutions of Fig. 1, and the star at $280 \mathrm{~K}$ indicates the transition point for the chosen parameter path. The inset shows the fit parameters $v_{2}$ and $v_{\mathrm{s}}$ as functions of temperature, connected by lines as guides to the eye. The values of the hopping parameter $\delta$ (using $t_{0}=1 / \Omega$ [6]) obtained from the extended MCT fits shown in Fig. 1 are also included in the inset and are close to the values reported in Ref. [1]. The critical temperature $T_{\mathrm{c}}=280 \mathrm{~K}$ is marked by a vertical line.

and above $400 \mathrm{GHz}$ to relaxational processes for lower frequencies. If a Markovian approximation to the spectra held below the microscopic excitations, then a white noise susceptibility, $\chi_{\text {white }}^{\prime \prime} \propto \omega$, should be observed. Such a white noise spectrum is shown in Fig. 1 with slope unity. Both steeper slopes, indicating oscillatory dynamics at high frequencies, and shallower slopes, indicating non-exponential low-frequency relaxation, appear in the spectra and in the fits. The possibility of choosing temperature independent oscillator parameters, even though $T$-dependence above $400 \mathrm{GHz}$ is apparent in the data, stresses that the mode-coupling effects can cause noticeable temperature variations of the low-frequency wing of the microscopic excitations. An anomalous increase of the structural relaxation spectra above the white noise approximation is predicted in asymptotic MCT solutions by the critical power law $\chi^{\prime \prime}(\omega) \propto \omega^{a}$. It is exhibited by the theoretical curve labeled c in Fig. 1 and its observation is expected to mark the temperature closest to $T_{\mathrm{c}}$. The critical exponent $a$ follows from $\lambda$ and equals $a=0.312$ for OTP.

The $\alpha$-relaxation peak, which lies in the experimental frequency window for temperatures around the melting temperature $T_{\mathrm{m}}$, can be characterized by a $T$-independent Kohlrausch stretching parameter $\beta=0.79$ [1]. The $\alpha$-peak is fitted by the schematic model solution which asymptotically exhibits a von Schweidler power law wing, $\chi^{\prime \prime}(\omega) \propto \omega^{-b}$. The exponent $b=0.582$ also follows from $\lambda$. From the detailed analysis of Ref. [1] one can also conclude, as is corroborated by our fits, that the enhancement of the spectrum above a white noise background in the minimum region of the spectra cannot be explained by a simple interpolation between a white noise wing of the microscopic spectrum and a stretched $\alpha$-process.

In the solutions of the schematic model shown in Fig. 1, the asymptotic idealized MCT predictions carry corrections due to the microscopic processes and due to finite distances of the control parameters from the bifurcation. In atomic glass formers, additional thermally activated processes are present and lead to corrections to the idealized MCT picture. In the frequency range of the minimum in $\chi^{\prime \prime}(\omega)$, these corrections are understood in asymptotic expansions [6]. The activated processes included in the extended MCT explain the existence of the $\alpha$-process below $T_{\mathrm{c}}$ and the systematic increase of the spectra at low frequencies and temperatures above the fits with the schematic model. The analysis of the low temperature spectra with the asymptotic predictions of the extended MCT from Ref. [1] is redone in Fig. 1 using our slightly different value of $\lambda$ and thus somewhat different control parameters. In this analysis one further $T$-dependent parameter $\delta$ enters characterizing the thermally activated processes $[3,6]$; it is 
also shown in Fig. 2. Close to the critical temperature, at $T=280 \mathrm{~K}$, the schematic model solution and the extended MCT asymptotic fit both exhibit the critical power law and overlap for about half a decade in frequency. For lower temperatures, the corrections included in the schematic model, which mask the critical power law towards higher frequencies, and the corrections to $\chi^{\prime \prime} \propto \omega^{a}$ due to the thermally activated processes, which come in from lower frequencies, limit the window for the asymptotic results of the idealized MCT.

\section{Conclusions}

The universal asymptotic results of the MCT predict a sensitive dependence of the structural dynamics on the experimental control parameters like temperature. If corrections to the asymptotic results are large it is difficult to identify the predicted scaling laws. In this case it would be best to use microscopically formulated MCT equations to quantitatively calculate non-asymptotic corrections [7]. As a compromise, schematic MCT models may be used to test the applicability of the MCT to more complicated systems, where microscopic MCT equations are yet unknown. The present study of GHz spectra of OTP demonstrates this approach and indicates that all observed features of the structural relaxation of OTP can be described by smooth temperature dependence of two model parameters. Including thermally activated processes below $T_{\mathrm{c}}$ a complementary fit using the extended MCT covers the spectra at lower temperatures and matches to the schematic model fit. The values of the MCT exponents and of the critical temperature which we find are in reasonable agreement with previous neutron [4] and light scattering studies [1].

\section{Acknowledgements}

This work was supported by NATO Collaborative Research Grant No. CRG-930730 and by Verbundprojekt BMBF 03-G04TUM.

\section{References}

[1] H.Z. Cummins, G. Li, W.M. Du, Y.H. Wang, G.Q. Shen, Prog. Theor. Phys. 126 (1997) 21; H.Z. Cummins, Y.H. Wang, G. Li, W.M. Du, W. Losert, G.Q. Shen, J. NonCryst. Solids (submitted).

[2] A. Patkowski, W. Steffen, G. Meier, E.W. Fischer, J. NonCryst. Solids 172-174 (1994) 52; W. Steffen, A. Patkowski, H. Glaser, G. Meier, E.W. Fischer, Phys. Rev. E49 (1994) 2992.

[3] W. Götze, L. Sjögren, Rep. Prog. Phys. 55 (1992) 241.

[4] A. Tölle, H. Schober, J. Wuttke, F. Fujara, Phys. Rev. E56 (1997) 809.

[5] T. Franosch, W. Götze, M.R. Mayr, A.P. Singh, Phys. Rev. E55 (1997) 3183.

[6] W. Götze, L. Sjögren, Z. Phys. B65 (1987) 415; M. Fuchs, W. Götze, S. Hildebrand, A. Latz, J. Phys.: Condens. Matter 4 (1992) 7709.

[7] T. Franosch, M. Fuchs, W. Götze, M.R. Mayr, A.P. Singh, Phys. Rev. E55 (1997) 7153. 